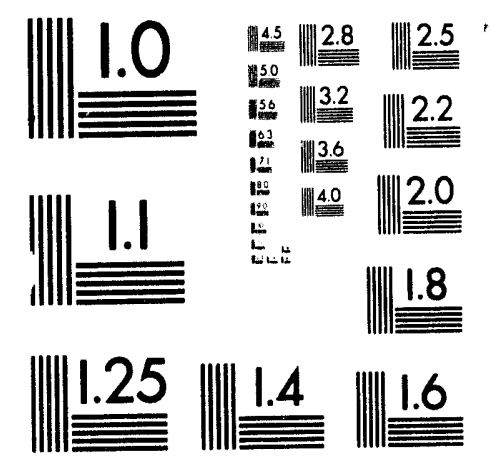



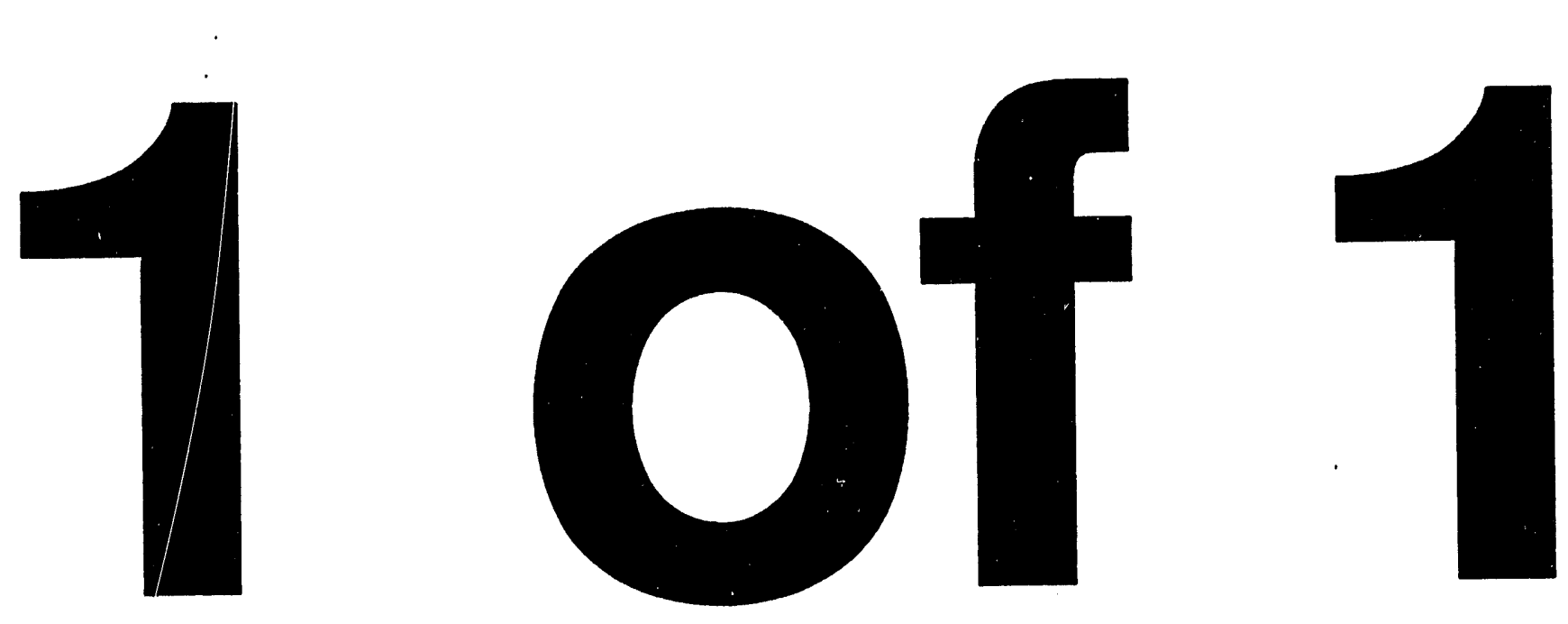


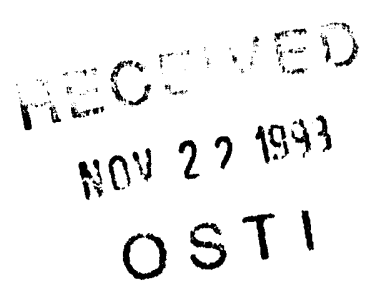

\title{
A STORAGE RING BASED INVERSE COMPTON SCATTERING ANGIOGRAPHY SOURCE?
}

\author{
E. B. Blum
}

September, 1993

\section{DISCLAIMER}

This report was prepared as an account of work sponsored by an agency of the United States Government. Neither the United States Government nor any agency thereof, nor any of their employees, makes any warranty, express or implied, or assumes any legal liability or responsibility for the accuracy, completeness, or usefulness of any information, apparatus, product, or process disclosed, or represents that its use would not infringe privately owned rights. Reference herein to any specific commercial product, process, or service by trade name, trademark, manufacturer, or otherwise does not necessarily constitute or imply its endorsement, recommendation, or favoring by the United States Government or any agency thereof. The views and opinions of authors expressed herein do not necessarily state or reflect those of the United States Government or any agency thereof.

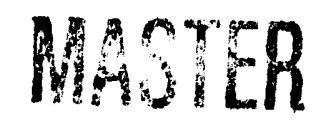




\title{
A Storage Ring Based Inverse Compton Scattering Angiography Source?*
}

\author{
E.B. Blum \\ National Synchrotron Light Source \\ Brookhaven National Laboratory \\ Upton, NY 11973
}

Producing the $33.17 \mathrm{KeV}$ photons required for coronary angiography with synchrotron radiation requires a combination of a high energy storage ring with an extremely high field wiggler. Such a source may be too big and expensive to be installed in even the largest medical center. Something other than synchrotron radiation may be needed for a practical source. $^{1}$

Inverse Compton scattering has been used in the LEGS experiment at NSLS ${ }^{2}$ and elsewhere to produce high energy photons. In this process, a head on collision between a low energy photon and a high energy electron transfers energy to the photon which is then emitted in approximately the direction of the incoming electron. For a given electron energy, more higher energy photons can be produced by this method than by synchrotron radiation. This suggests that inverse Compton scattering can possibly be used for a low cost angiography source. The prospects for such a source will be examined in this paper. Unfortunately, the results will show that although it is easy to obtain the required photon energy, an extremely complicated source will be needed to produce the required number of photons.

Some requirements for an angiography source are listed in Table 1. They are based on the chracteristics of the X-17 angiography $\mathrm{x}$-ray beam at the NSLS. The required intensity, $2 \times 10^{8}$ photons $/\left(.5 \mathrm{~mm}^{2}\right)-4 \mathrm{msec}-70 \mathrm{eV}$, is an estimate of what is required to produce a clinical quality image.

In a head on collision between a high energy electron and a low energy photon ${ }^{3}$, the photon emerges at an angle $\theta$ to the incident electron direction, with energy

$$
E_{\gamma}=\frac{4 \gamma^{2} \epsilon_{L}}{1+\gamma^{2} \theta^{2}+4 \gamma \epsilon_{L} /\left(m c^{2}\right)},
$$

where $\gamma$ is the Lorentz factor for the incident electron, $\epsilon_{L}$ is the energy of the incident photon ( $L$ for laser, the presumed source of the photons), and $m c^{2}$ is the rest energy of the electron.

1. This report is a summary of an accelerator physics seminar presented at the NSLS on May 28, 1992.

2. C.E. Thorn, et. al., Nucl. Instr. and Meth. A285 (1989) 447-458.

3. Ibid.

* This work was supported by the U.S. Department of Energy. 
The highest energy photons are emitted with $\theta=0$, at energy

$$
E_{C}=\frac{4 \gamma^{2} \epsilon_{L}}{1+4 \gamma \epsilon_{L} /\left(m c^{2}\right)},
$$

called the Compton edge. This shows that $33.17 \mathrm{KeV}$ photons can be produced in a collision between $417.9 \mathrm{MeV}$ electrons and photons with a wavelength of $100 \mu \mathrm{m}$. The photon beam contains all energies from 0 to $E_{C}$, distributed with differential cross-section

$$
\frac{d \sigma}{d E_{\gamma}}=\frac{2 \pi r_{0}^{2} \epsilon_{L}}{E_{\gamma}^{2} y^{2}}\left(\frac{2 \gamma}{1+x^{2}}\right)^{2}\left[y+\frac{1}{y}-\left(\frac{2 x}{1+x^{2}}\right)^{2}\right],
$$

where $r_{0}=2.82 \times 10^{-15} \mathrm{~m}$ is the classical electron radius,

$$
x=\theta \gamma=\left[4 \gamma \epsilon_{L}\left(\frac{\gamma}{E_{\gamma}}-\frac{1}{m c^{2}}\right)-1\right]^{1 / 2},
$$

and

$$
y=1+\frac{4 \gamma \epsilon_{L}}{\left(1+x^{2}\right) m c^{2}} .
$$

Fig. 1 shows the differential cross section for the scattering of $100 \mu \mathrm{m}$ photons off $417.9 \mathrm{MeV}$ electrons. The total (Thompson) cross section,

$$
\sigma_{T}=\int_{0}^{E_{C}} \frac{d \sigma}{d E_{\gamma}} d E_{\gamma}=665 \mathrm{mb},
$$

is independent of $\epsilon_{L}$ and $E_{\gamma}$.

In a collision between a bunched electron beam and the light from a pulsed laser, photons are produced in an energy band $\Delta E$, near the Compton edge, at a rate

$$
R=\sigma_{E_{C}} \Delta E N_{L} F_{e},
$$

where $\sigma_{E c}$ is the differential cross section at the Compton edge, $N_{L}$ is the number of laser photons per pulse, and $F_{e}$ is the electron flux. It is assumed that the shape of the incident photon and electron bunches is the same in all three spatial dimensions and that the divergence of the incident beams is small compared to the divergence of the emergent photons, as given by eqn. (1) and $\Delta E$. Eqns. $1-5$ show that

$$
\sigma_{E c} \approx \frac{4 \pi r_{0}^{2}}{E_{c}}
$$

is independent of the incident photon and electron energies. The number of laser photons per pulse

$$
N_{L}=\frac{E_{L}}{\epsilon_{L}}=\frac{\lambda_{L} E_{L}}{h c},
$$


where $E_{L}$ is the total energy of the laser pulse and $\lambda_{L}$ is the laser wavelength. In practice, $E_{L}$ is roughly independent of the laser wavelength. Hence, the photons are produced at a rate

$$
R=\Delta E_{L} \frac{\lambda_{L} E_{L} F_{e}}{h c}
$$

that is proportional to the wavelength of the incident photons. This tends to drive the inverse Compton scattering source to long wavelength incident photons. However, eqn. 2 shows that the electron beam energy

$$
E_{e} \approx m c^{2} \sqrt{\frac{\lambda_{L} E_{c}}{4 h c}}
$$

increases as the square root of the incident photon energy, and with it the size and expense of the source. Together, eqns. 10 and 11 favor a source with an electron beam energy in the range from ten to a few hundred $\mathrm{MeV}$ and incident infrared photons.

The high energy electron beam needed in the angiography source can be supplied by either a storage ring or an accelerator such as a linac or betatron. In a storage ring, the circulating electron bunch has many opportunities to interact with the laser during the time required for the x-ray exposure. This provides a high effective flux $F_{e}$ with relatively few electrons compared to the single use of a beam from an accelerator.

An example of the type of small storage ring that might be used in an angiography source was provided by the $x$-ray lithography source (XLS) project at the NSLS. Table 2 shows the properties of a $417.9 \mathrm{MeV}$ storage ring that uses $100 \mu \mathrm{m}$ photons to produce 33.17 $\mathrm{KeV}$ photons at the Compton edge. The storage ring parameters are suggested by the XLS design but the required specifications for emittance and coupling, although reasonable, are in no way similar to XLS.

The use of a storage ring as the electron beam source requires a laser with a repetition rate equal to the ring bunch frequency. The combination of laser pulse repetition rate, pulse energy, and wavelength required by the storage ring design can not be provided by a conventional laser. Instead, a free electron laser (FEL) driven by the electron beam from a superconducting linac was chosen as the source of the photons. The FEL parameters are listed in Table 3. The FEL is configured as a ring laser with the inverse Compton scattering taking place within the FEL cavity as shown in Fig. 2. Energy is recovered from the electrons after they pass through the FEL undulator by redirecting the beam through the linac.

The design of the FEL is the most uncertain part of the source. The linac beam power, repetition rate, and peak current needed have never been achieved simultaneously. Although it may be possible to build, the FEL would be complicated and at or beyond the state of the art. It does not in any way appear to be suitable for a hospital based angiography source. In choosing to use inverse Compton scattering as a source of $33 \mathrm{KeV} x$-rays, the size and expense of a high energy storage ring were exchanged for the expense and complexity of an FEL with an extremly high repetition rate. Storage rings still look like a better source of high energy photons. 
Table 1

Angiography Requirements

Fundamental Requirements:

$\begin{array}{lcl}\text { X-ray Energy } & 33.17 & \mathrm{KeV} \\ \text { Energy Spread } & 70 & \mathrm{eV} \\ \text { Distance to Source } & 40 & \mathrm{~m} \\ \text { Exposure Time } & 4 & \mathrm{msec} \\ \text { Horizontal Beam Size at Patient } & 12 & \mathrm{~cm} \\ \text { Pixel Size } & 0.5 \mathrm{~mm} \times 0.5 \mathrm{~mm}\end{array}$

Derived Requirements:

Horizontal Divergence

Vertical Divergence

$3 \mathrm{mrad}$ (from distance to source and horizontal size)

Intensity

$2 \times 10^{8}$ photons $/\left(0.5 \mathrm{~mm}^{2}\right)-4 \mathrm{msec}-70 \mathrm{eV}$

By Comparison:

$X-17$ Intensity

(Bragg monochromator)

$3 \times 10^{7}$ photons $/\left(0.5 \mathrm{~mm}^{2}\right)-4 \mathrm{msec}-70 \mathrm{eV}$

Table 2

Storage Ring Characteristics

Suggested by XLS Design:

Energy

Current

Number of Bunches

417.9 $\mathrm{MeV}$

$350 \quad \mathrm{~mA}$

Revolution Frequency $35 \quad \mathrm{MHz}$

Requirement:

Emittance

Coupling

$0.25 \mathrm{~mm}-\mathrm{mrad}$

0.10

At Interaction Point:

$\sigma_{\mathbf{x}^{\prime}}$

$\sigma_{y^{\prime}}$

$\sigma_{x}$

$\sigma_{y}$

Electron Flux

$2.09 \times 10^{25} \mathrm{~m}^{-2} \mathrm{sec}^{-1}$

3 mrad (requirement)

$0.13 \mathrm{mrad}$ (requirement)

$0.083 \mathrm{~mm}$ (from required divergence and emittance)

$0.20 \mathrm{~mm}$ (from required divergence and emittance)

Requires from FEL:

Wavelerigth

Laser Energy/Pulse

Repetition Rate

Average Power

100

543

105

57

$\mu \mathrm{m}$

$\mu \mathrm{J}$

$\mathrm{MHz}$

$\mathrm{KW}$ 
Table 3

FEL Characteristics

Undulator:

Period

K

Peak Field

Number of Periods

Length

Electron Beam:

Energy

Average Current

Beam Power

Repetition Rate

Electrons/Bunch

Bunch Length

Peak Current

Beam Radius

$\begin{array}{cc}10 & \mathrm{~cm} \\ 1.414 & \\ 1.5 & \mathrm{KG} \\ 4 & \\ 40 & \mathrm{~cm}\end{array}$

$\begin{array}{cl}16.16 & \mathrm{MeV} \\ 50.4 & \mathrm{~mA} \\ 814 & \mathrm{KW} \\ 105 & \mathrm{MHz} \\ 3 \times 10^{9} & \\ 1 & \mathrm{~mm} \\ 144 & \mathrm{~A} \\ 2.5 & \mathrm{~mm}\end{array}$

Optics:

Number of Mirrors

Reflectivity

Total Loss

Rayleigh Length

0.99

0.04

4

$20 \mathrm{~cm}$

Small Signal FEL Model:

Efficiency

Gain

Power

0.13

0.10

102

KW 


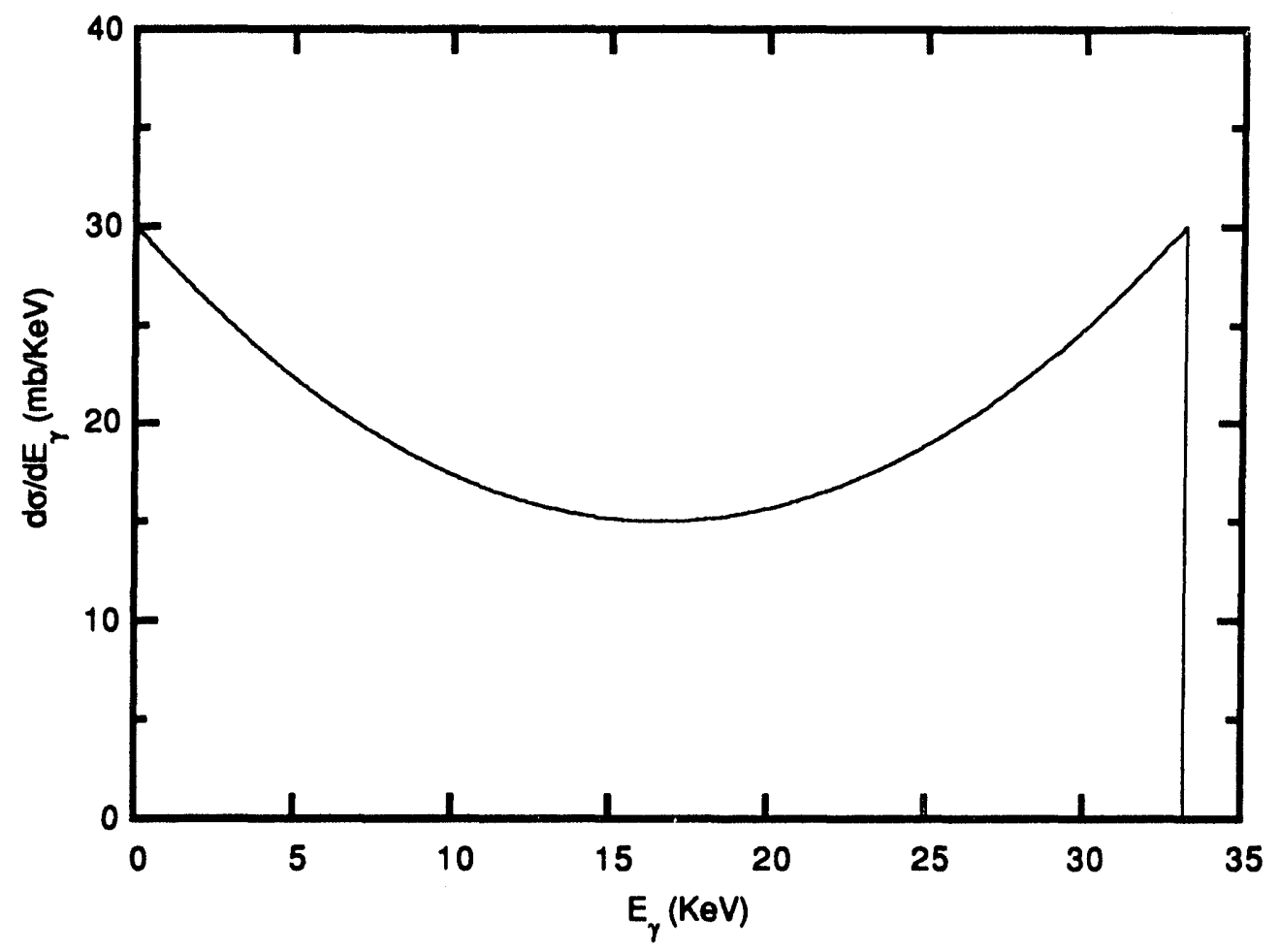

Fig. 1. Differential cross section for inverse Compton scattering, $E_{c}=33.17 \mathrm{KeV}$. 


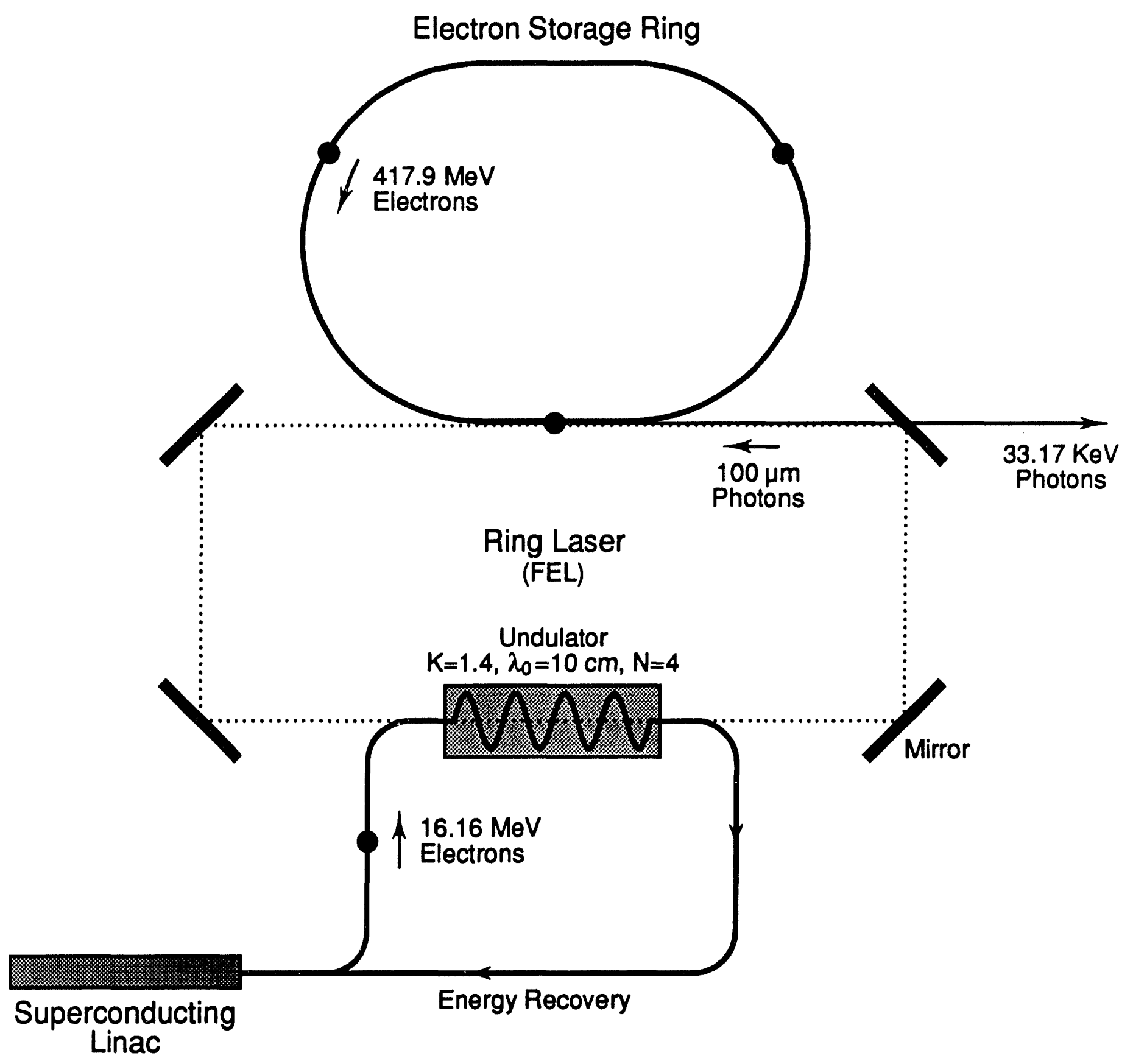

Fig. 2. Schematic view of inverse Compton scattering $\mathrm{x}$-ray source. 
